\title{
List of posters
}

Posters are available on-line at http://www.astro.keele.ac.uk/iaus256/proceedings, either as fully edited/indexed manuscripts, numbered P-xx, or PDF files, numbered PDF-xx.

Colour - magnitude diagrams of candidate age-gap filling LMC clusters . . . . . PDF-1 E. Balbinot, B.X. Santiago, L.O. Kerber, B. Barbuy, B.M.S. Dias

Age and metallicity of globular clusters in the SMC from integrated spectra ... PDF-2 B. Barbuy, B.M.S. Dias, P. Coelho, T.E.P. Idiart, L.O. Kerber

HE 0437-5439: The first hypervelocity star from the LMC . . . . . . . . . . PDF-3 A.Z. Bonanos, A.Z.M. López-Morales, I. Hunter, R. Ryans

LHA 115-S 23, from B[e] to A[e]: A highly precessing system or a chemically peculiar

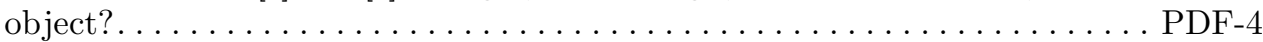
M. Borges Fernandes, M. Kraus, J. Kubát, F.X. de Araújo

A Spitzer IRAC census of the asymptotic giant branch populations in Local Group

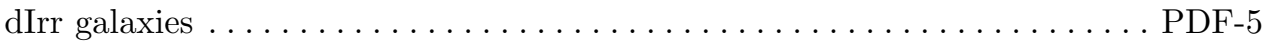
M.L. Boyer, E.D. Skillman, J.Th. van Loon, D.C. Jackson, R.D. Gehrz

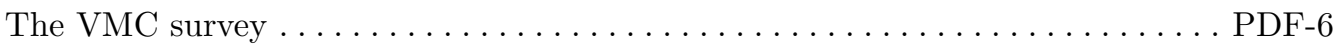
M.-R.L. Cioni, K. Bekki, G. Clementini, W.J.G. de Blok, J. Emerson, C.J. Evans, R. de Grijs, B.K. Gibson, L. Girardi, M.A.T. Groenewegen, V.D. Ivanov, R. Napiwotzki, M. Marconi, C. Mastropietro, B. Moore, T. Naylor, J.M. Oliveira, V. Ripepi, J.Th. van Loon, M.I. Wilkinson, P.R. Wood

LMC PNe: Spitzer IR luminosity functions and AGB halos. . . . . . . . . . PDF-7 M. Cohen, J.L. Hora, Q.A. Parker, W.A. Reid

Self-consistent physical parameters for Magellanic Cloud clusters from ColourMagnitude Diagram modelling: Application to SMC clusters observed with

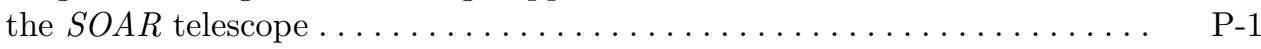
B.M.S. Dias, L.O. Kerber, B.X. Santiago, B. Barbuy

New radio-continuum surveys of the Magellanic Clouds. . . . . . . . . . . PDF-8 M.D. Filipović, E.J. Crawford, A. Hughes, H. Leverenz, A.Y. De Horta, J.L. Payne, L. Staveley-Smith, J. Dickel, F.H. Stootman, G.L. White

Multi-wavelength observations of Magellanic SNRs . . . . . . . . . . PDF-9 M.D. Filipović, J.L. Payne, F. Haberl, W. Pietsch, P.F. Winkler, E.J. Crawford, A.Y. Horta, F.H. Stootman

Formation and evolution process of giant molecular clouds in the Large Magellanic

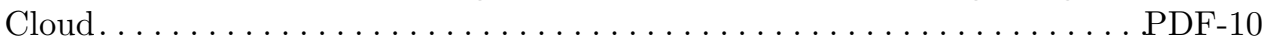

Y. Fukui, M. Murai, H. Iritani, A. Kawamura, T. Wong, Y. Mizuno,

E. Muller, N. Mizuno, T. Ohnishi, L. Staveley-Smith, S. Kim

Turbulence: A probe of the dynamics and physics of the Magellanic Stream .... $\mathrm{P}-2$ I. Goldman

The Magellanic System X-ray sources . . . . . . . . . . . . . . P A.J. Gosling, S.A. Farrell, N.A. Webb, J.J.E. Kajava 
Star-forming regions in the Magellanic Clouds . . . . . . . . . . . . . PDF-11

D.A. Gouliermis, Th. Henning, W. Brandner, A.E. Dolphin, S. Schmeja,

R.A. Gruendl, Y.-H. Chu, R.S. Klessen

Main-sequence fitting ages for a large sample of LMC clusters . . . . . . . . PDF-12

A.J. Grocholski, A. Sarajedini, C.L. Macone, A.A. Cole, D. Geisler,

V.V. Smith

Galactic C and S stars as guidelines for Magellanic Cloud AGB stars . . . . . . P P-4 R. Guandalini, M. Busso

Be/X-ray binaries in the SMC seen with XMM-Newton. . . . . . . . PDF-13 F. Haberl, P. Eger

The detection of ammonia $\left(\mathrm{NH}_{3}\right)$ in the $\mathrm{LMC} \ldots \ldots \ldots \ldots \ldots \ldots \ldots \ldots \ldots \ldots \ldots$ C. Henkel, J. Ott

Molecular clouds in the H II region N 11 in the Large Magellanic Cloud. . . . . . PDF-15 C. Herrera Contreras, M. Rubio, A.D. Bolatto

Properties of LMC molecular clouds from MAGMA, the Magellanic Mopra

Assessment . . . . . . . . . . . . . . . . . . . . PDF-16

A. Hughes, T. Wong, E. Muller, J.L. Pineda, J. Ott, The MAGMA

Collaboration

Neutrino-electron scattering in hot magnetic fields in the Magellanic System with

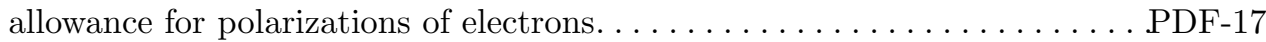

V.A. Huseynov, R.E. Gasimova

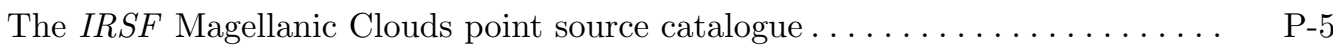

D. Kato

Study of molecular clouds and star formation in the Magellanic Clouds by

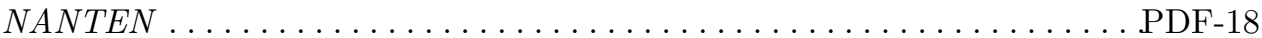

A. Kawamura, Y. Mizuno, M. Murai, T. Minamidani, N. Mizuno, T.

Onishi, E. Muller, A. Mizuno, M.D. Filipović, L. Staveley-Smith, S. Kim,

M. Rubio, Y. Fukui

SkyMapper: Mapping the Magellanic Clouds . . . . . . . . . . . . . . . PDF-19

S.C. Keller, G. Da Costa, K. Freeman, M.S. Bessell, P. Francis,

B. Schmidt, P. Tisserand

The VISTA survey of the Magellanic Clouds: Unveiling their star formation

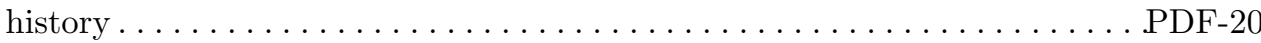

L.O. Kerber, L. Girardi, S. Rubele, M.-R.L. Cioni

Photometric metallicities of star clusters in the Large Magellanic Cloud . . . . . . P P-6

A. Kučinskas, V. Dobrovolskas, A. Černiauskas, T. Tanabé

The trigger of the AGB superwind: The importance of carbon . . . . . . . P

E. Lagadec, A.A. Zijlstra

Dark clouds in the Small Magellanic Cloud. . . . . . . . . . . . . . . . . . . . P P-8

M.-Y. Lee, S. Stanimirović, J. Ott, A.D. Bolatto, J.Th. van Loon,

K.E. Devine, J.M. Oliveira, P.A. Jones, M.R. Cunningham 
Spectroscopic and infrared properties of Type II Cepheids in the LMC and the

Galaxy......................... P-9

Lloyd Evans, K.R. Pollard

Chemical evolution of the Magellanic Clouds based on planetary nebulae .... . P-10 W.J. Maciel, R.D.D. Costa, T.E.P. Idiart

The source and formation of the Magellanic Stream. . . . . . . . . . . PDF-21 S.R. Majewski, D.L. Nidever, W. Butler Burton

Inventoring aromatic emission in the Magellanic Clouds . . . . . . . . . PDF-22

A.R. Marble, C.W. Engelbracht, C. Bot, K.D. Gordon, J.L. Hora, Spitzer SAGE Team

Zero-age main-sequence rotational velocities of Be/Oe stars and luminous GRB progenitors in the Magellanic Clouds . . . . . . . . . . . . . P-11

C. Martayan, J. Zorec, Y. Frémat

Variability of B and Be stars in the LMC/SMC: Binaries and pulsations. . . . . P P-12 C. Martayan, P. Diago, J. Gutiérrez-Soto, J. Fabregat, A.-M. Hubert, M. Floquet, C. Neiner, M. Mekkas

A search for intracluster dust of stellar clusters in the LMC . . . . . . . . . PDF-23 N. Matsunaga

The global gas and dust budget of the Large Magellanic Cloud: AGB stars and supernovae, and impact on the ISM $\ldots \ldots \ldots \ldots \ldots \ldots \ldots \ldots \ldots \ldots \ldots \ldots \ldots$ M. Matsuura

Spitzer survey of the Large Magellanic Cloud: Surveying the Agents of a Galaxy's

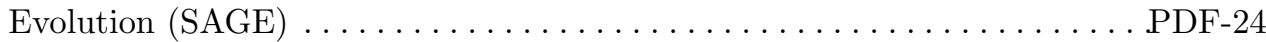

M. Meixner, M. Sewilo, U.P. Vijh, C. Leitherer, B.A. Whitney, M. Meade,

B.L. Babler, R. Indebetouw, J.L. Hora, K.D. Gordon, C.W. Engelbracht, M. Block, K.A. Misselt, R.D. Blum, W. Reach, J.-P. Bernard, A.G.G.M. Tielens, Spitzer SAGE Team

HERschel Inventory of The Agents of Galaxy Evolution (HERITAGE) in the

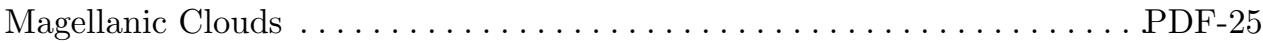

M. Meixner, S. Madden, S. Hony, A.G.G.M. Tielens, K.D. Gordon, A.D. Bolatto, C.W. Engelbracht, E. Churchwell, B.L. Babler, T. Beck, J.-P. Bernard, C. Bot, F. Boulanger, S. Bracker, G.C. Clayton, M. Cohen, K. Dobashi, Y. Fukui, F. Galliano, J. Harris, J.L. Hora, A. Hughes, R. Indebetouw, F. Israel, A. Kawamura, S. Kim, C. Kramer, A. Li, K. Long, M. Marengo, F. Markwick-Kemper, M. Matsuura, M. Meade, K.A. Misselt, E. Muller, D. Neufeld, A. Nota, S. Oey, K. Okumura, J.M. Oliveira, T. Onishi, D. Paradis, A. Poglitsch, W. Reach, T. Robitaille, M. Rubio, D. Rubin, M. Sauvage, M. Sewilo, L.J. Smith, S. Srinivasan, S. Stanimirović, J.Th. van Loon, B.A. Whitney, M. Wolfire, Herschel HERITAGE Team

Variability parameters and mass-loss tracers in the LMC . . . . . . . . PDF-26 M. Meixner, D. Riebel, S. Srinivasan, O. Fraser, B.L. Babler, M. Block, S. Bracker, C.W. Engelbracht, K.D. Gordon, J.L. Hora, R. Indebetouw, C. Leitherer, M. Meade, K.A. Misselt, B.A. Whitney, M. Sewilo, U.P. Vijh 
ISM in the LMC from Spitzer/SAGE and extinction data. . . . . . . . . PDF-27 M. Meixner, J.-P. Bernard, D. Paradis, W. Reach, K. Dobashi, A. Hughes,

A. Kawamura, Spitzer SAGE Team

LMC: Stellar populations and their gradients . . . . . . . . . . .

I. Meschin, C. Gallart, A. Aparicio, R. Carrera, M. Monelli, S.L. Hidalgo, P.B. Stetson

Sub-mm observations of GMCs in the SMC with NANTEN2 . . . . . . PDF-28

N. Mizuno, Y. Mizuno, M. Rubio, F. Bertoldi, J.L. Pineda, F. Boulanger,

M. Vidal, A. Kawamura, E. Muller, M. Murai, T. Minamidani, T. Onishi,

Y. Fukui, J. Stutzki, U. Klein, M.G. Burton, B.C. Koo, A. Bentz,

NANTEN2 Consortium

Sub-mm observations of molecular clouds in the N 159 region with the NANTEN2

telescope .......................... P-15

Y. Mizuno, N. Mizuno, A. Kawamura, T. Ohnishi, Y. Fukui, T.

Minamidani, J. Stutzki, J.L. Pineda, U. Klein, F. Bertoldi, B.C. Koo, M.

Rubio, M.G. Burton, J. Ott, NANTEN2 Consortium, Spitzer SAGE Team

Massive binaries in Magellanic star clusters. . . . . . . . . . . . . . . PDF-29

N. Morrell, P. Massey, K. deGioia-Eastwood, L. Penny, D. Gies, N. Melena,

B. Skiff

Characterizing the low-mass molecular component in the Northern Small

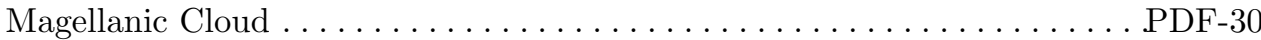

E. Muller, J. Ott, A. Hughes, J.L. Pineda, T. Wong, N. Mizuno, A.

Kawamura, Y. Mizuno, Y. Fukui, T. Onishi

Modeling mass-loss and infrared excess in Large Magellanic Cloud Cepheids ... P-16 H.R. Neilson, C.-C. Ngeow, S.M. Kanbur, J.B. Lester

An $\mathrm{H} \alpha$ giant shell in the South-East of the Large Magellanic Cloud. . . . . . . . PDF-31 M. Oddone, A. Laval, E. LeCoarer, G. Goldes

Mid-infrared spectrum variation of long-period variables in the Large Magellanic

Cloud.......................... P-17

K. Onaka, T. Kamizuka, I. Sakon, T. Miyata, T. Tanabé, Y. Okada,

I. Yamamura

Radio planetary nebulae in the Magellanic Clouds . . . . . . . . . . . PDF-32

J.L. Payne, M.D. Filipović, W.C. Millar, E.J. Crawford, A.Y. Horta,

F.H. Stootman, D. Urošević

Multiphase PC/PL relations: Comparison between theory and observations . . PDF-33

V. Ripepi, S.M. Kanbur, M. Marconi, C.-C. Ngeow, M. Turner, I. Musella

STEP survey - the SMC in time: Evolution of a prototype interacting dwarf

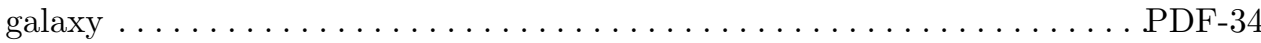

V. Ripepi, M. Marconi, I. Musella, M. Dall'Ora, A. Ruoppo, M. Capaccioli,

G. Clementini, M. Tosi, G. Macario, D. Romano, M. Cignoni, E. Brocato,

G. Raimondo, M. Cantiello, M.-R.L. Cioni, A. Nota, M. Sirianni, J.S.

Gallagher, E.K. Grebel, E. Cappellaro

Preliminary results of the SUNY Oswego IR LMC survey . . . . . . . . . . PDF-35

F. Ripple, S.M. Kanbur, L. Macri, C.-C. Ngeow, S. Nikolaev, T. Barnes 
Stellar rotation rate in the Magellanic Clouds: Does metallicity matter? . . . . . PDF-36 F. Royer, P.L. North, C. Melo, J.R. de Medeiros, E.K. Grebel, J.-C.

Mermilliod, B.L. Canto Martins, C. Martayan, A. Maeder

The VISTA survey of the Magellanic Clouds: Image simulations and expected

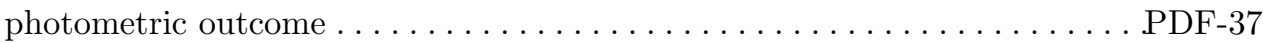

S. Rubele, L. Girardi, L.O. Kerber, M.-R.L. Cioni

Star formation in the Magellanic Bridge . . . . . . . . . . . . . PDF-38 M. Rubio, K. Rübke, N. Mizuno

Spatial motion of the Magellanic Clouds — tidal models ruled out? . . . . . . PDF-39 A. Rüžička, A. Theis, J. Palouš

Merger candidates among star clusters in the Small Magellanic Cloud. . . . . . PDF-40 J.F.C. Santos Jr., A.A. Schmidt, E. Bica

Quantitative spectroscopy of BA-type supergiants in the SMC: Basic atmospheric parameters and the flux-weighted gravity-luminosity relationship. . . . . P-18 F. Schiller, N. Przybilla, R.-P. Kudritzki, M.A. Urbaneja

Photometric variations in LMC planetary nebulae $\ldots \ldots \ldots \ldots \ldots \ldots \ldots$ PDF-41 R.A. Shaw, A. Rest, G. Damke

A panchromatic view of Magellanic star formation: From optical to infrared. . PDF-42 L.J. Smith, L.R. Carlson, K. Romita, M. Sewilo, E. Sabbi, M. Meixner, T. Robitaille, A. Nota, B.A. Whitney, K.D. Gordon

Infrared excess emission from asymptotic giant branch stars in the Large Magellanic Cloud. . . . . . . . . . . . . . . . . . . . . . . . PDF-43 S. Srinivasan, M. Meixner, C. Leitherer, U.P. Vijh, K. Volk, R.D. Blum, B.L. Babler, M. Block, S. Bracker, M. Cohen, C.W. Engelbracht, K.D. Gordon, J. Harris, J.L. Hora, R. Indebetouw, F. Markwick-Kemper, M. Meade, K.A. Misselt, M. Sewilo, B.A. Whitney

Near-infrared spectroscopy of SAGE sources in the LMC . . . . . . . . . . PDF-44 S. Srinivasan, K. Volk, R.D. Blum

Modelling the circumstellar disks of Be stars in X-ray binary systems in the SMC

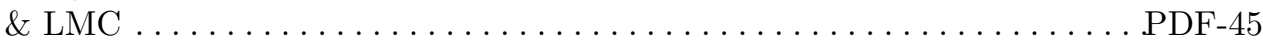
L.J. Townsend

Identification of post-AGB stars from the SAGE database . . . . . . . . PDF-46 T. Ueta, A.G. Ginsburg, Spitzer SAGE Team

Variable sources in the LMC using the SAGE survey. . . . . . . . . . . . PDF-47 U.P. Vijh, M. Meixner, B.L. Babler, M. Block, S. Bracker, C.W. Engelbracht, B.-Q. For, K.D. Gordon, J.L. Hora, R. Indebetouw, C. Leitherer, M. Meade, K.A. Misselt, M. Sewilo, S. Srinivasan, B.A. Whitney

Detailed abundances in star clusters and field stars as probes of the chemical

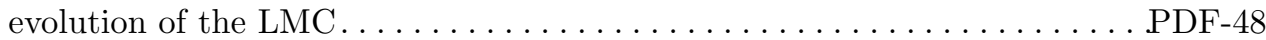
S. Villanova, D. Geisler, R. Mateluna, F. Mauro, A.A. Cole, A.J. Grocholski, A. Sarajedini, V.V. Smith 
A three-decade outburst of the LMC luminous blue variable R 127 draws to a close P-19 N.R. Walborn

Abundances and isotope ratios in $\mathrm{N} 113$. PDF-49

M. Wang, Y.-N. Chin, C. Henkel, J.B. Whiteoak, M.R. Cunningham

AGB variables in the Fornax dwarf galaxy and the Large Magellanic Cloud . . PDF-50 P.A. Whitelock, J.W. Menzies, M.W. Feast

The correlation between $\mathrm{CO}$ and $\mathrm{H}$ I emission in the Large Magellanic Cloud . PDF-51 T. Wong, Y. Fukui, A. Kawamura, N. Mizuno, A. Hughes, J. Ott, E. Muller, J.L. Pineda, L. Staveley-Smith, S. Kim, Y. Mizuno, M. Murai

Exploring the SMC to the faintest X-ray fluxes: First results from a Chandra

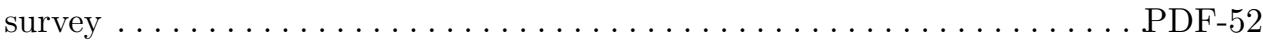
A. Zezas, S. Laycock, J. Hong, V. Antoniou, C. Heinke, V. Kalogera, J. McDowell, J. Drake, J.S. Gallagher, M. Smith, G. Fabbiano 